\title{
Stenting to prevent esophageal stricture after circumferential endoscopic submucosal dissection: an experimental study
}

\section{(ㄷ)(1)}

Authors

Jan Martinek*, 1,2,3,4, Radek Dolezel*,2,6, Bara Walterova², Marek Kollar ${ }^{2,4,5}$, Stefan Juhas², Jana Juhasova², Zuzana Vackova ${ }^{1,4}$, Rastislav Hustak ${ }^{1}$, Jakub Erben ${ }^{7}$

Institutions

1 Institute for Clinical and Experimental Medicine, Hepatogastroenterology, Prague, Czech Republic

2 Czech Academy of Sciences, Institute of Animal Physiology and Genetics, PIGMOD, Libechov, Czech Republic

3 University of Ostrava Faculty of Medicine, Department of Clinic Subjects, Ostrava, Czech Republic

4 Charles University, First Faculty of Medicine, Institute of Physiology, Prague, Czech Republic

5 Institute for Clinical and Experimental Medicine, Clinical and Transplant Pathology Centre, Prague, Czech Republic

6 Charles University, Department of Surgery, 2nd Faculty of Medicine Central Military Hospital, Prague, Czech Republic.

7 Technical University of Liberec, Faculty of Textile Engineering, Department of Nonwovens and Nanofibrous Materials, Liberec, Czech Republic

submitted 10.12 .2019

accepted after revision 11.5.2020

Bibliography

Endoscopy International Open 2020; 08: E1698-E1706

DOI 10.1055/a-1261-3103

ISSN 2364-3722

(c) 2020. The Author(s).

This is an open access article published by Thieme under the terms of the Creative Commons Attribution-NonDerivative-NonCommercial License, permitting copying and reproduction so long as the original work is given appropriate credit. Contents may not be used for commecial purposes, or adapted, remixed, transformed or built upon. (https://creativecommons.org/licenses/by-nc-nd/4.0/)

\section{Corresponding author}

Prof. Jan Martinek, MD, PhD, AGAF, Department of

Hepatogastroenterology, IKEM, Videnska 1958, 14021

Prague 4, Czech Republic

Fax: + 420973202899

jan.martinek@volny.cz

\section{ABSTRACT}

Background and study aims Circular ESD (CESD) is a treatment option for patients with extensive early esophageal cancer. Its major drawback is the development of a stricture. Stenting may represent an attractive prevention strategy. We designed an experimental study to assess the effect of stents covered with acellular biomatrix $(A B)$ and a drug-eluting stent.

Materials and methods Thirty-five 35 pigs underwent CESD and were randomized into six groups: $G 1$ (control), G2 (SEMS), G3 (SEMS + AB), G4 (SEMS + AB + steroid-eluting layer), $G 5$ (biodegradable stent $[B D]), G 6(B D+A B)$. SEMS were placed alongside the post-CESD defect, fixed and removed after 21 days. The main outcomes were stricture development, severity, and histopathology.

Results Pigs with BD stents $(G 5,6)$ experienced severe inflammation and hypergranulation without biodegradation, therefore, these groups were closed prematurely. Significant strictures developed in 29 of 30 pigs (96.7\%). The most severe stricture developed in G2 and G4 (narrowest diameter (mm) $8.5 \pm 3,3(\mathrm{G} 2)$ and $8.6 \pm 2.1$ (G4) vs. $17 \pm$ 7.3 (G1) and $13.5 \pm 8.3$ (G3); $P<0.01$. Signs of re-epithelization were present in $67 \%$ and $71 \%$ in G1 and G2 and in $100 \%$ in G3 and G4. The most robust re-epithelization layer was present in G4. The inflammation was the most severe in G1 (mean score 2.3) and least severe in G4 (0.4).

Conclusions Stenting did not effectively prevent development of post-CESD esophageal stricture. SEMS with AB resulted in improved re-epithelization and decreased stricture severity. Steroid-eluting SEMS suppressed inflammation. BD stents seem inappropriate for this indication.

* These authors contributed equally 


\section{Introduction}

Minimally invasive endoscopic resection techniques allow curative resection of early esophageal neoplasia. While endoscopic resection allows en-bloc removal of only lesions up to $15 \mathrm{~mm}$, endoscopic submucosal dissection (ESD) allows en-bloc removal of larger and even circumferential lesions. En-bloc resection allows complete histopathological staging, which is why ESD is a preferred resection technique in patients presenting with early esophageal neoplasia, especially those who have more aggressive squamous carcinoma [1]. If a lesion is completely removed (R0 resection) and histopathological assessment confirms a low risk of generalization, endoscopic treatment is considered curative and patients do not have to undergo riskier esophagectomy.

Esophageal neoplastic lesions, especially squamous, can spread extensively and even around the whole esophageal circumference with their invasion remaining superficial. Thus, extensive ESD comprising more than $75 \%$ of the circumference may be required. Extensive ESD is technically feasible and safe if performed by an experienced endoscopist [1]. Its major limitation is a high risk of stricture formation. If the resection comprises $60 \%$ of the esophageal circumference, the risk of stricture is $70 \%$ to $80 \%$ and basically $100 \%$ if the resection is circumferential [2]. Post-ESD (or post-widespread endoscopic resection) strictures are often refractory and require several sessions of endoscopic therapy [3]. Such a complex treatment increases the risk of adverse events (AEs) and decreases patient quality of life and may delay subsequent oncological treatment, if necessary.

Because of the high risk of stricture formation, a preventive strategy should be considered in all patients undergoing circumferential or near-circumferential esophageal ESD [3]. Several such strategies have been tested in both experimental and clinical studies but none of them has ever proved to have a satisfactory preventive effect [4]. These strategies include systemic or local administration of steroids, endoscopic dilatation, stenting, and tissue engineering methods (cell-based therapies).

Esophageal stents are an attractive option because the technique is easy to master and stents are relatively inexpensive. Self-expanding metallic stents (SEMS) have reduced the risk of stricture formation by approximately $50 \%$ in a small randomized study [5]. Nevertheless, the evidence of SEMS effectiveness in prevention of post-ESD esophageal strictures is rather limited. Biodegradable (BD) stents delayed the time of severe stricture by 6 weeks but did not prevent stricture in one experimental study [6]. BD stents, if effective, would be the preferred option as they do not need to be removed. Covering a stent with various types of materials promoting re-epithelization and decreasing fibrosis, or drug-eluting stents, may be another option for increasing the effectiveness of stenting. Thus, we designed an experimental randomized study to assess the effect of SEMS and BD stents in preventing post-circumferential endoscopic submucosal dissection (CESD) esophageal stricture in an animal model. In particular, we aimed to: (1) assess the effect of a covering of the stents with acellular biomatrix $(A B)$ from a pig's epidermis (which has been shown to promote re-epithelialization and accelerate healing process in skin wounds [7, 8]); (2) assess the effect of drug-eluting SEMS (covered by both $A B$ and a nanofibers based layer eluting steroids); and (3) assess the effect of $B D$ stents (w/o $A B$ ). We tested the hypothesis that the local action of $A B$ and/or steroids may prevent post-CESD strictures and improve re-epithelization and overall healing quality.

\section{Material and methods}

The study was approved by the Animal Protection Committee (Authorisation No.7779/ 2004-1020) in compliance with applicable legal regulations (No.246/1992, 207/ 2004). All experiments were carried out according to the guidelines for the care and use of experimental animals and approved by the Resort Professional Commission of the CAS for Approval of Projects of Experiments on Animals (Approved protocol No.27/ 2016, 16/2017, 39/2018).

\section{Animals and the study protocol}

Thirty-five experimental pigs (Libechov breed, Czech Academy of Sciences) were randomized into six groups according to prevention type: group $G 1$ (control, $n=6$ ), G2 (SEMS, $n=8$ ), G3 $(S E M S+A B, n=8), \quad G 4$ (SEMS + AB + steroid carrier, $n=8), \quad G 5$ $(B D, n=3), G 6(B D+A B, n=2)$. ( Table 1). After CESD, all pigs received an intramuscular injection of metylprednisolone acetate (Depo-Medrol, Pfizer Manufacturing N.V., Puurs, Belgium) for 30 days (a low depot dose $-4.3 \mathrm{mg} / \mathrm{kg}$ every 3 days (equivalent to $30 \mathrm{mg}$ of oral prednisolone) and the dose was tapered by $20 \%$ weekly. We administered the corticoid intramuscularly (oral administration would have been technically challenging) to all pigs because it mirrored the usual clinical practice, in which patients after extensive ESD are given systemic steroids.

\section{CESD}

CESD was performed under general anesthesia with $1.5 \%$ isoflurane (Piramal Healthcare, Morpeth, England) and fentanyl (Ratiopharm GmbH, Ulm, Germany) in the mid-esophagus and its length was $5 \mathrm{~cm}$. We used a single-channel endoscope (EVIS EXERA II GIF H180 J, diameter 9.9 mm; Olympus, Tokyo, Japan) and Dual Knife (KD-650L; Olympus, Tokyo, Japan). After mark-

\begin{tabular}{|l|l|l|}
\hline \multicolumn{1}{|l|}{ Table $\mathbf{1}$} & Study groups. \\
\hline Group no. & No. of pigs & Prevention type \\
\hline 1 & 6 & None \\
\hline 2 & 8 & SEMS \\
\hline 3 & 8 & SEMS + biomatrix \\
\hline 4 & 8 & SEMS + biomatrix + steroid \\
\hline 5 & 3 & BD stent \\
\hline 6 & 2 & BD stent + biomatrix \\
\hline SEMS, self-expandable metallic stent; BD, biodegradable stent.
\end{tabular}


ing the upper and lower edges and performing submucosal injection, circular incisions were done at both edges and then, alternating submucosal injection and dissection, CESD was completed, and the specimen was removed en-bloc. After CESD, no antibiotics were administered. The post-CESD esophagus is shown in > Fig. 1.

\section{Stenting}

A SEMS or a BD stent was placed in groups G2-6 (> Fig. 1). In G2-G4, a 10-cm fully-covered SEMS (FCSEMS) (Wallflex Prox CVD, $25 \mathrm{~mm}$, Boston Scientific, Natick, Massachusetts, United States - G2, G3) or (ELLA-CS, Hradec Kralove, Czech Republic, G4) were deployed under endoscopic control to ensure a precise stent position overlapping the whole defect ( $\mathbf{F i g . 1 b}$ ). In G5 and G6, we used 10-cm uncovered BD stents (BD ELLA-CS, Hradec Kralove, Czech Republic) and the deployment was also controlled endoscopically.

Stents were fixed using a lasso technique. Two polyamide threads were attached to the oral stent margin and pulled out through the nostrils and were anchored to a piercing made through the septum between the nostrils.

\section{Stent covering}

Stents in the G3, G4, and G6 groups were covered with an acellular biomatrix (Xe-Derma, Medicem, Prague, Czech Republic). $A B$ is made from piglet dermis and contains $3 D$ collagenous and elastic scaffolding fibers. This biomaterial has been proven to stimulate proliferation of keratinocytes through expression of p63, involucrin, and CD29, providing a high-quality, multilayer epithelization of superficial skin layers in pigs without any immunologic rejection [7-9]. Such a biomatrix is clinically approved in the treatment of burns or diabetic foot ulcers as a temporary skin cover [7-9].

We created a cylinder of biomatrix by suturing the prefabricated $A B$ sheets with Vicryl (2-0, Ethicon; Conelia, Georgia, United States) and coupled it with the aboral stent end ( $>$ Fig.2). Prior to stent insertion, the biomatrix was moistened with saline to become supple and hydrophilic.

Stents in $\mathrm{G} 4$ were also covered with a drug-eluting layer. We used an FCSEMS prototype (ELLA-CS, Hradec Kralove, Czech Republic) with a three-layer construct consisting of a Nitinol stent (25-mm diameter and 100-mm length), AB - (Xe-Derma) layer with a thickness of $150 \mu \mathrm{m}$ and the third layer made from polydioxanone (PDO) nanofibers containing $100 \mathrm{mg}$ prednisone (250- $\mu \mathrm{m}$ thickness, > Fig. 3 and > Fig.4). Drug-eluting stents (including covering with $\mathrm{AB}$ ) were made by ELLA, Hradec Kralove, Czech Republic.

\section{Post-CESD regime}

After recovery, the pigs received fluids without restrictions and restarted feeding with a special half-liquid diet (quick rice porridge with yogurt) for 2 to 3 days and then they restarted a standard feeding pattern.

A control endoscopy (to check for a stricture in G1 or to exclude a stent migration in G2 to G6 was performed approximately 2 weeks after the procedure). SEMS were removed 21 days after CESD and the pigs were followed up and the next

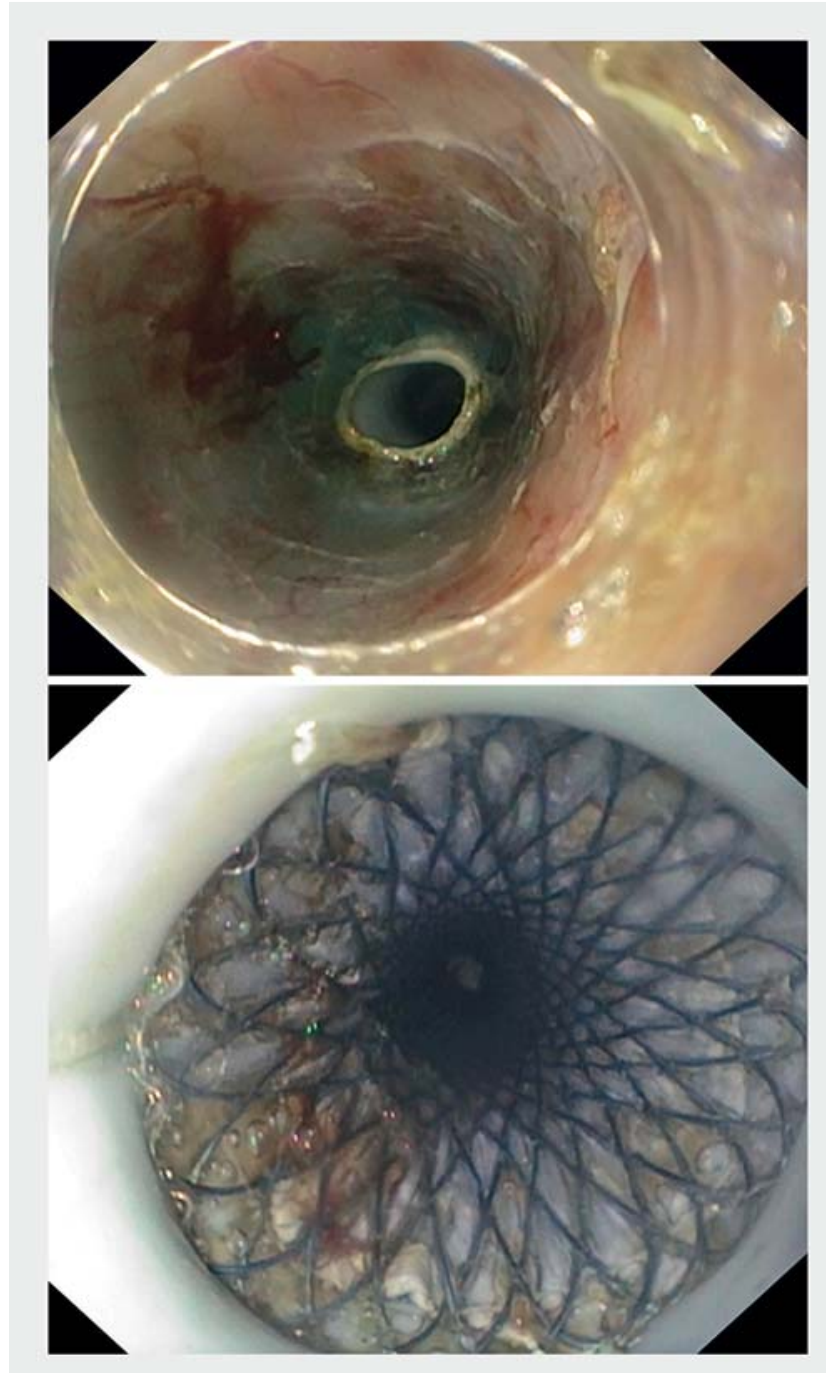

- Fig. 1 a Esophagus after CESD. b SEMS deployed.

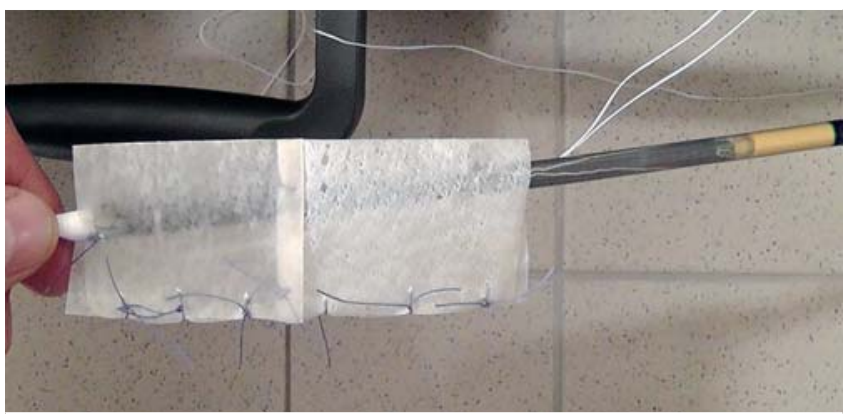

- Fig. 2 SEMS covering process with biomatrix (G3).

endoscopy was scheduled for postoperative Day 40 (G2-G6) or 30 (G1), or if any symptoms of a stricture appeared (vomiting, feeding problems).

In the case of symptoms suggestive of a stricture, the pigs underwent endoscopy and if a stricture non-passable with $r$ an endoscope was found, they were euthanized and underwent necropsy. The esophagus with resection area (stricture) was 


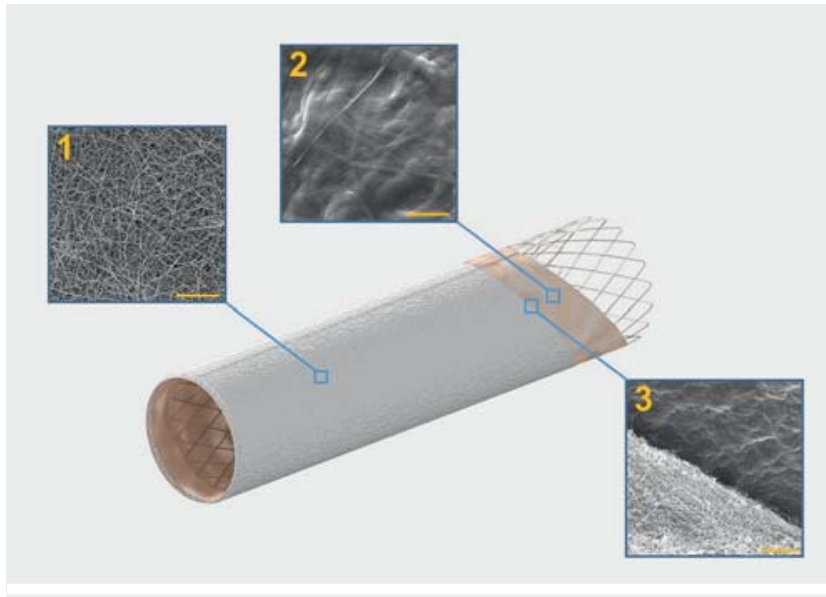

Fig. 3 The scheme of the drug-eluting stent: 1 - nanofibrous layer, 2 -Biomatrix (Xe-Derma) (scale bars: $50 \mu \mathrm{m}$ ), 3-nanofibrous layer/biomatrix overlay (scale bar: $200 \mu \mathrm{m}$ ).
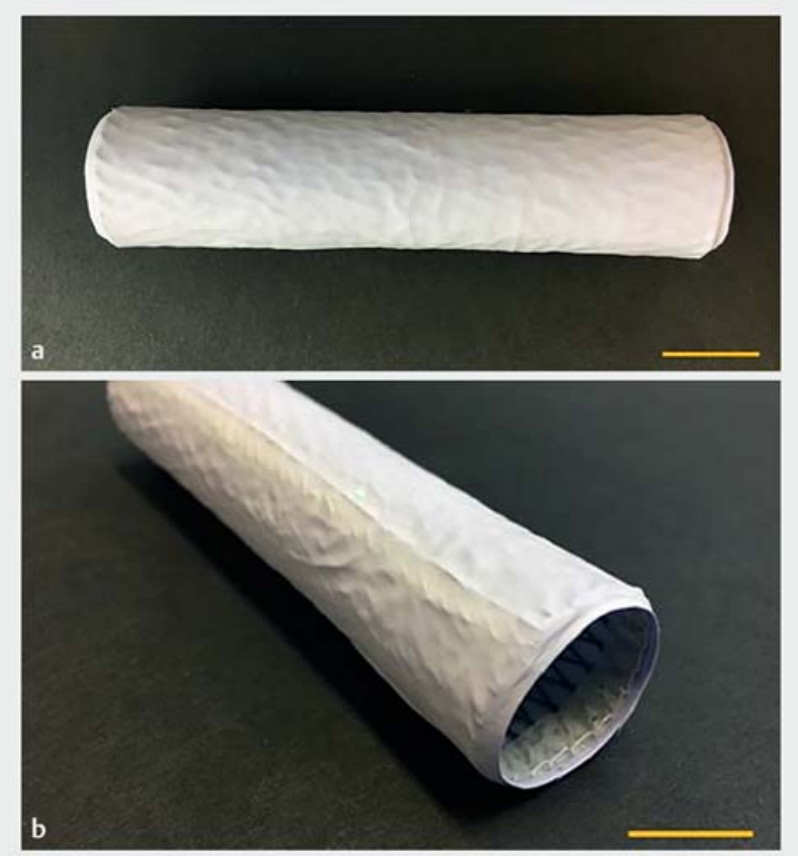

Fig. 4 Drug-eluting SEMS with $A B$ and drug-eluting layers, scale bar $2 \mathrm{~mm}$.

taken out, cut along the longitudinal axis and various stricture parameters were measured ( $\vee$ Fig. 5). Subsequently, the specimen was fixed in formalin and sent for a histopathological analysis.

\section{Histopathology}

All specimens were assessed by one blinded gastrointestinal pathologist. Sections were taken from the normal mucosa, transition zone between the lesion (stricture) and normal epithelium, and the stricture and cut into $5-\mathrm{mm}$ slices. Then, material was embedded into paraffin, cut into 3- to 4- $\mu \mathrm{m}$ sections, stained

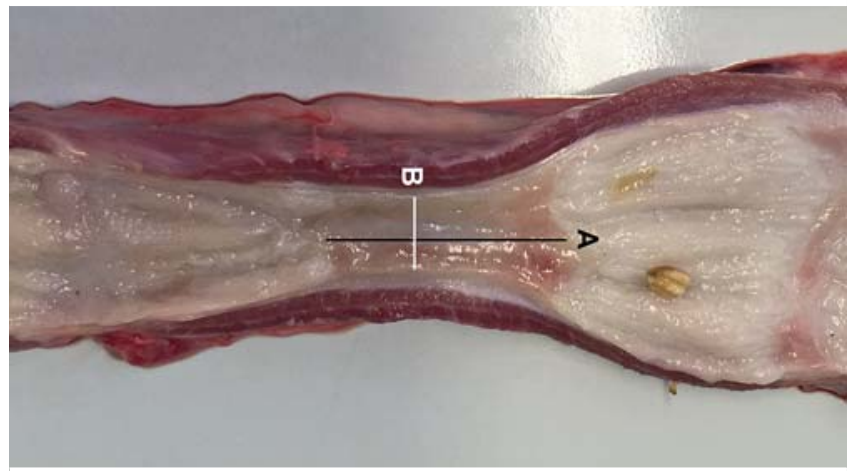

- Fig. 5 Parameters of stricture severity. a Length. b Narrowest lumen width.

with hematoxylin-eosin (H\&E) and Sirius red with elastics for evaluation of fibrosis.

\section{Outcomes}

The main outcome was development of an endoscopically nonpassable stricture (using an endoscope with a 9.9-m diameter). Secondary outcomes were stricture severity (length of the stricture, the narrowest width in the sheared esophagus on autopsy, > Fig.5) and time to stricture formation (from CESD in G1, G5, G6, or from SEMS removal in G2, G3, G4).

Histopathological outcomes were re-epithelialization, fibrosis thickness, and severity of inflammation. All microscopic parameters were assessed by using IES $1200 \mathrm{D}$ software attached to a microscope (Nikon, Eclipse Cl-L, Tokyo, Japan). All samples were blindly evaluated by an experienced pathologist (MK).

Sections were taken from normal mucosa including resection margin, transition zone, and post-CESD defect (processed completely) and cut into $5-\mathrm{mm}$ slices. The material was then embedded in paraffin blocks and cut into 3- to 4- $\mu \mathrm{m}$ sections and stained with H\&E and Sirius red with elastics (to measure fibrosis thickness).

Re-epithelialization was described by length and width. The length was defined as the longest distance of the newly formed epithelium from the transitional zone (border of the normal epithelium and newly formed epithelium). The width was defined as the thickest part of the newly formed epithelium.

Microscopic inflammation was evaluated semi-quantitatively (0-none, 1 -mild, 2 -intermediate, 3 -severe). Level 0 was defined as normal/usual presence of inflammatory cells, level 1 was set as a mild inflammation limited to the mucosa; level 2 was defined as moderate inflammatory infiltration reaching lamina muscularis propria; level 3 was defined as fibrinous purulent inflammation with ulceration or presence of micro abscesses.

\section{Statistical analysis}

Data are presented as means with standard deviation or as proportions with percentages. The categorical data were analyzed by using Fisher's exact test and continuous data were analyzed with one-factor analysis of variance (ANOVA) test and the Stu- 

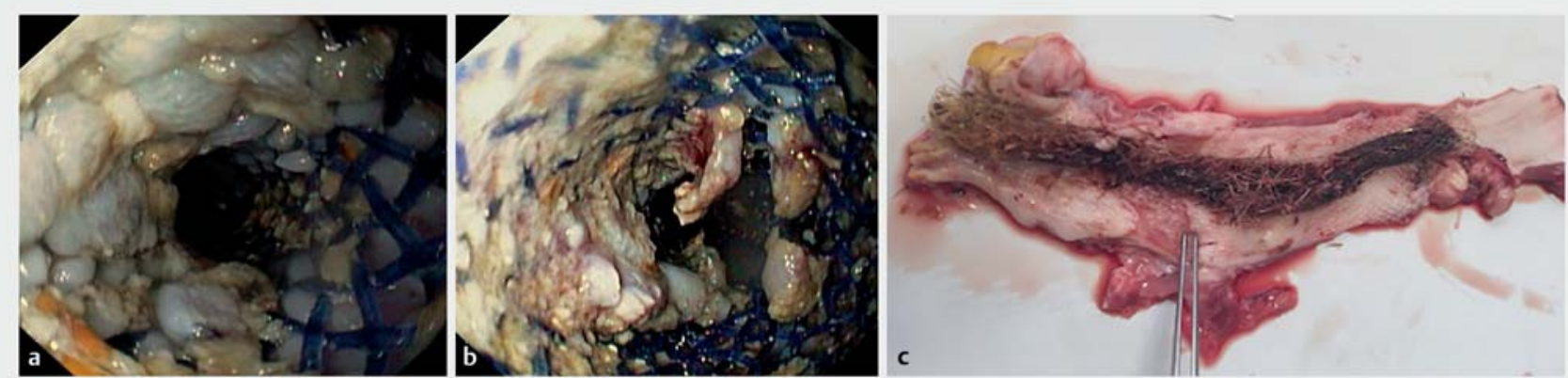

- Fig. 6 CESD with BD stent placement. a, b Endoscopic views. c Necropsy.

dent's $t$-test. A Bonferroni correction was applied in the case of a multiple comparison. $P<0.05$ was considered significant.

\section{Results}

\section{CESD and stenting}

A total of 35 CESDs were performed, the mean procedure time was $58 \pm 13.9$ minutes, and there were no significant complications such as bleeding or perforation. The mean length of CESD defect was $55 \pm 3.0 \mathrm{~mm}$. All stents were successfully inserted and deployed in a correct position and no migration occurred. In G2 to G4, all SEMS were successfully removed 3 weeks after CEMS and no residual $A B$ or nanofiber layer was present on the stent outer surface.

\section{Biodegradable stents}

All the pigs with BD stents $(G 5, G 6)$ experienced severe inflammation and massive hypergranulation (along the stent and particularly at both proximal and distal ends) causing stenosis and food stagnation inside the stent while biodegradation had not occurred even 3 weeks after CESD ( $\bullet$ Fig. $\mathbf{6}$ ). Thus, we decided to stop enrollment into these groups prematurely and the pigs that had already undergone the procedure were excluded from the final analysis ( $\bullet$ Table 1 ).

\section{Strictures}

Significant strictures developed in all but one pig from G1 (29/ 30, 96.7\%). Strictures developed $13.5 \pm 6$ days after CESD (G1) and $14.1 \pm .5$ days (G2), $15.3 \pm 6$ days (G3) and $12.5 \pm 3.7$ days (G4) after stent removal, $P>0.1$. The only pig without a stricture (G1) was euthanized 30 days after CESD.

\section{Stricture severity}

The macroscopic parameters are summarized in $\downarrow$ Table 2 .

The most severe stricture developed in G2 and G4 (narrowest diameter $[\mathrm{mm}] 8.5 \pm 3,3(\mathrm{G} 2)$ and $8.6 \pm 2.1$ (G4) vs. $17 \pm 7.3$ $(G 1)$ and $13.5 \pm 8.3(G 3) ;(P<0.01)$ ( $>$ Fig. 7$)$.

Stricture length did not differ among the groups even though strictures trended to be longer in G1 compared with other groups $(P=0.15)$.
- Table 2 Macroscopic outcomes.

\begin{tabular}{|c|c|c|c|}
\hline $\begin{array}{l}\text { Group } \\
\text { no. }\end{array}$ & $\begin{array}{l}\text { Stricture } \\
\text { presence }\end{array}$ & $\begin{array}{l}\text { Narrowest width } \\
(\mathrm{mm})\end{array}$ & $\begin{array}{l}\text { Length of stricture } \\
(\mathrm{mm})\end{array}$ \\
\hline $1^{1}$ & $5 / 6,83 \%$ & $17 \pm 7.3^{2}$ & $21.6 \pm 19.2^{3}$ \\
\hline 2 & $8 / 8,100 \%$ & $8.5 \pm 3.3$ & $14.6 \pm 8.6$ \\
\hline 3 & $8 / 8,100 \%$ & $13.5 \pm 8.3^{2}$ & $15.2 \pm 11$ \\
\hline 4 & $8 / 8,100 \%$ & $8.6 \pm 2.1$ & $17 \pm 10.3$ \\
\hline \multicolumn{4}{|c|}{$\begin{array}{l}\text { Data are presented as means } \pm \text { standard deviation. } \\
{ }^{1} \text { The pig without a stricture was not included into the analysis of width and } \\
\text { length. } \\
2 P<0.01 \mathrm{G} 1, \mathrm{G} 3 \text { vs. G2, G4. } \\
{ }^{3} \text { Stricture length in G1 vs. G2 to G4; P= } 0.15 \text {. }\end{array}$} \\
\hline
\end{tabular}

\section{Microscopic outcomes}

Histopathological outcomes are summarized in $>$ Table 3 and $\checkmark$ Table 4.

Re-epithelization was present in all animals with $A B$ (groups $G 3, G 4)$. In groups without $A B$, re-epithelization was less frequent (G1-67\%, G2-75\%, $P=0.07$, > Table 3).

Length of re-epithelization (measured as the longest distance from the edge of the defect toward a new grown top of re-epithelization, $>$ Fig. 8 ) did not differ among the groups ( $P=$ $0.13)$. The widest re-epithelisation layer was found in G4 (236 \pm $202 \mu \mathrm{m})$, which was significantly more compared to other groups, $P=0.01$.

The thickness of fibrosis was similar among the four groups, $P=0.67$. Microscopic inflammation was least severe in G4 and G3 ( Table 4). No pig had severe fibrinous purulent inflammatory reaction in $G 4$ and $G 3$, while it was present in some pigs in the remaining groups ( $\bullet$ Table 4 ).

\section{Discussion}

In the present study we have shown that placement of a SEMS, either BD or metallic, with or without a covering by an acellular biomatrix from pig epidermis, did not prevent development of an esophageal stricture after circumferential ESD in pigs. A steroid-eluting stent (SEMS+AB+drug-eluting layer), tested for the first time in this indication, did not prevent the stricture 

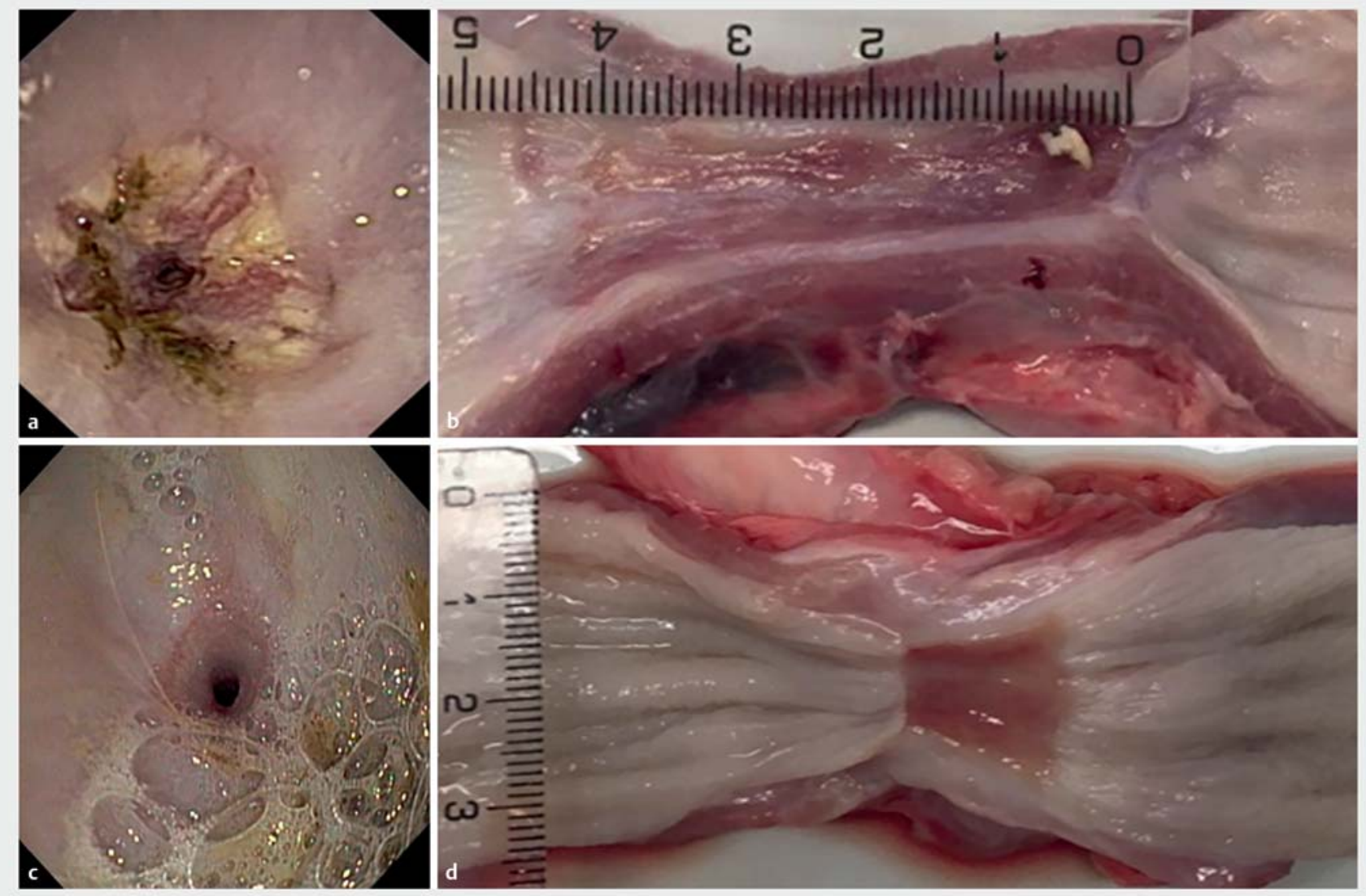

Fig. 7 a, b Macroscopic appearance of post-CESD strictures in the control group c, $\mathbf{d}$ and in the group with SEMS covered with biomatrix (G3). Stricture in the control group is longer compared to G3.

either. Covering the stent with the biomatrix resulted in decreased stricture severity and better quality of healing.

Use of a steroid-eluting stent significantly decreased the severity of inflammation but did not influence stricture severity. A BD stent, either with or without biomatrix, did not prevent post-CESD stricture and its use was accompanied by severe inflammatory reaction and hypergranulation, resulting in stent occlusion while biodegradation did not occur. The problem with severe hyperplastic tissue reaction causing tissue ingrowth with subsequent stenosis has been described in several reports with $B D$ stents, especially if stent biodegradation lasted longer [10]. The fact that a BD stent itself does not prevent post-CESD stricture has already been demonstrated in another experimental study [6], but the authors did not experience problems with BD stents as severe as was the case in our study (no biodegradation, severe hypergranulation, secondary stent occlusion by food). We decided to stop enrollment into both experimental groups with BD stent prematurely as we considered it unethical to continue. Our results show that BD stents seem inappropriate in this indication. There are no clinical studies examining the effectiveness of BD stents in prevention of post-CESD strictures except for the two human cases treated successfully with the PLLA BD esophageal stent in Japan [11].
The risk of esophageal stricture is the major drawback of extensive circumferential or near-circumferential esophageal ESD. The risk of stricture is high $(70 \%$ to $80 \%$ ) if more than three-quarters of the circumference is resected, or when the whole circumference is removed, then the rate of stricture is practically $100 \%[12]$.

These strictures are often difficult to treat, requiring several sessions of endoscopic dilatation, further increasing the risk of complications and decreasing patients quality of life.

Several preventive strategies have been tested in clinical or experimental studies, none of which, unfortunately, have gained general acceptance as a preventive method of choice. The best evidence is available for systemic or local administration of steroids even if they are unable to prevent stricture formation in patients with circular ESD - but if treated with steroids, these strictures are less severe and easier to treat [13-18].

Stents are an attractive option because they are widely available, and their use is relatively easy and less expensive, compared to other preventive strategies such as tissue engineering methods. FCSEMS have been tested in small clinical or experimental studies but their use has not been generally accepted. Ye et al. [19] treated 23 patients with a CESD and all patients received a FCSEMS; a stricture developed in four of 23 patients $(17 \%)$, which was significantly less than an expected stricture 
> Table 3 Microscopic parameters.

\begin{tabular}{|c|c|c|c|c|}
\hline Group no. & Re-epithelization presence & $\begin{array}{l}\text { Re-epithelization length } \\
(\mu \mathrm{m})^{1}\end{array}$ & $\begin{array}{l}\text { Width of new epithelial } \\
\text { layer }(\mu \mathrm{m})\end{array}$ & Fibrosis thickness $(\mu \mathrm{m})^{2}$ \\
\hline 1 & $4 / 6,67 \%$ & $669 \pm 533$ & $98 \pm 89$ & $2992 \pm 1530$ \\
\hline 2 & $6 / 8,75 \%$ & $886 \pm 1107$ & $86 \pm 89$ & $2700 \pm 721$ \\
\hline 3 & $8 / 8,100 \%$ & $924 \pm 755$ & $137 \pm 70$ & $2310 \pm 1093$ \\
\hline 4 & $8 / 8,100 \%$ & $679 \pm 479$ & $236 \pm 202^{3}$ & $2090 \pm 581$ \\
\hline \multicolumn{5}{|c|}{$\begin{array}{l}\text { Data are presented as means } \pm \text { standard deviation. } \\
1 \text { No significant difference among groups; } P=0.13 \text {. } \\
{ }^{2} \text { The thickness of fibrosis was similar among the groups; } P=0.67 \text {. } \\
{ }^{3} \text { The most robust width in } \mathrm{G} 4 \text { vs. } \mathrm{G} 1-3 ; \mathrm{P}=0.01 \text {. }\end{array}$} \\
\hline
\end{tabular}

- Table4 Microscopic inflammation severity.

\begin{tabular}{|c|c|c|c|c|}
\hline Group no. & $\begin{array}{l}\text { No. of pigs with } \\
\text { Score } 0\end{array}$ & $\begin{array}{l}\text { No. of pigs with } \\
\text { Score } 1-2\end{array}$ & $\begin{array}{l}\text { No. of pigs with } \\
\text { Score } 3\end{array}$ & Mean inflammation score ( \pm SD) \\
\hline 1 & $0(0 \%)$ & $4(67 \%)$ & $2(33 \%)$ & $2,3 \pm 0.5$ \\
\hline 2 & $0(0 \%)$ & $7(88 \%)$ & $1(12 \%)$ & $2.2 \pm 0.4$ \\
\hline 3 & $2(25 \%)$ & $6(75 \%)$ & $0(0 \%)$ & $1.3 \pm 0.9$ \\
\hline 4 & $5(63 \%)$ & $3(37 \%)$ & $0(0 \%)$ & $0.4 \pm 0.5^{1}$ \\
\hline
\end{tabular}

rate of $100 \%$. Wen et al [5] found that the proportion of patients who developed a stricture was significantly lower in the group with SEMS (18.2\%) than in the control group $(72.7 \%)$. This is the only randomized trial examining the effect of stenting in preventing post-ESD esophageal stricture.

In our experimental study, SEMS did not prevent stricture development. It is possible that having stents remain in place for 3 weeks is not enough and delayed stent withdrawal would have been more effective. However, even after 3 weeks, there were signs of granulation at both stent margins and we could not leave the stents in any longer, as tissue ingrowth and severe granulation would have occurred and therefore, stent withdrawal would have been difficult or near to impossible. In humans, stents may remain in the esophagus as long as 2 to 3 months without serious problems. That was also the reason why we administered systemic steroids to all pigs to eventually decrease inflammatory and hyperplastic reaction following stent insertion. One of the reasons for earlier tissue proliferation might be that we used stents designed for humans, which may have been too large for a pig esophagus, which is narrower than a human esophagus. Stent margin might have caused more pressure on the mucosa and stimulated an earlier tissue reaction than would be expected in a human.

However, we have shown a beneficial effect of SEMS covering with a biomatrix, which is used to increase re-epithelization and accelerate healing in various skin defects (burns, acute wounds, etc.) [7-9]. The biomatrix has also been shown to prevent excessive granulations [8]. Unfortunately, stricture pre- vention was not achieved but we demonstrated that the local effect of $A B$ promoted re-epithelization, led to less severe strictures, and improved healing quality in terms of decreased inflammation. Two other experimental studies examined the effect of $A B[20,21]$. In one of them, a biomatrix covered a postESD defect and was fixed with endoclips (no stent was used). AB significantly prevented stricture formation, improved quality of healing, and promoted re-epithelization. The major shortcoming of this study was that ESD was not very extensive but only half-circumferential [20]. In the second study [21], delivery of a commercially available biological substrate from pig dermis on a stent did not, like in our study, prevent post-CESD stricture, but in contrast to our results, it did not influence re-epithelization or inflammation.

Another experimental study demonstrated that covering a stent with human amniotic membrane may prevent post noncircumferential ESD stricture even though this study is limited due to a very short duration ( 2 weeks) and details about stent withdrawal are missing [22].

We are the first to test the effect of a steroid-eluting stent in preventing post-CESD stricture in a controlled experimental study. The theory for this approach/ comes from clinical evidence showing that local steroid injection is an effective strategy, at least in some clinical trials [14-16]. Combining stenting with the local effect of a steroid (and also of $A B$ in our study) could, therefore, be an interesting and potentially effective strategy. Unfortunately, we were unable to demonstrate any major effect of this new stent. It did not prevent stricture and 

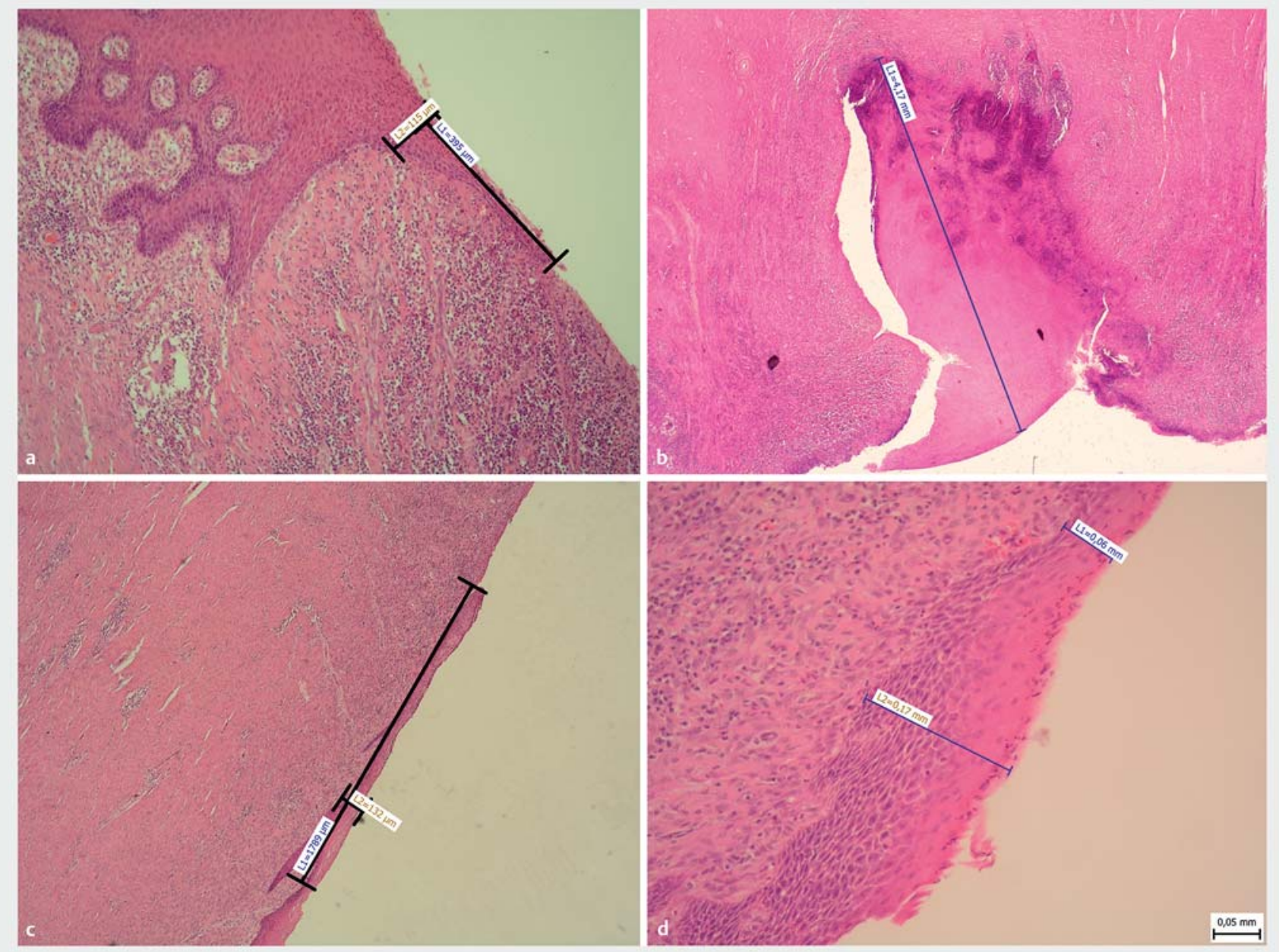

- Fig. 8 H\&E-stained microscopic images showing re-epithelization and inflammation a, $\mathbf{b}$ in the control group and $\mathbf{c}, \mathbf{d}$ the group G3 (SEMS with $A B$. Re-epithelization is longer and thicker in G3. (Magnification $40 \times A$ and C, $20 \times B, 100 \times D$ ).

it did not decrease stricture severity. However, inflammation in the post-CESD defect was absent or minimal.

Should the strategy of covering a stent by a BM or adding a drug-eluting layer be abandoned in clinical trials based on unsatisfactory results of several experimental studies, including our own? It might be the case that the pig model of CESD does not specifically mirror the effect of stenting in humans because, as mentioned earlier, stents cannot remain in a pig esophagus longer that 3 weeks, and therefore, produce more severe hypergranulation and tissue ingrowth. Furthermore, a model with CESD may be less appropriate to show a preventive effect of stenting (with any stent), less extensive ESD would have probably been more appropriate. We speculate that in our study, we managed to positively influence two of three main mechanisms of stricture development: influence on inflammation by steroids and on re-epithelialization by biomatrix. The remaining mechanism, overproduction of fibrotic tissue, was not inhibited in this study, therefore, it might prevail over the two factors.

Nevertheless, based on our "minor" positive effects of both BM and drug-eluting stent, we believe that further testing should be pursued in both experimental and clinical settings. One small case series showed rather promising results with a stent covered by triamcinolone-soaked polyglycolic acid sheet in patients after extensive esophageal ESD. No stricture occurred in patients with noncircumferential resection and stricture occurred in $50 \%(3 / 6)$ patients with circumferential resection [23].

Our study has several limitations: (1) the number of animals was relatively small; (2) covering the SEMS with biomatrix (G3) was done manually and not by a stent manufacturer; (3) two types of SEMS were used in our study (Wallflex and ELLA-CS), creating a kind of "technological bias" but as both types are $\mathrm{Ni}$ tinol-based, we do not think it influenced our results in a major way; and (4) no pharmacokinetic data regarding steroid release from a nanofiber-based layer are available.

\section{Conclusion}

In conclusion, we have shown that a SEMS covered with the acellular BM from pig dermis resulted in less severe post-CESD strictures with better re-epithelialization. Steroid-eluting SEMS 
did not prevent stricture or affect stricture severity but decreased inflammation to a minimum. Even though these stents did not prevent post-CESD stricture development in the experimental setting, SEMS covering with acellular scaffolds and steroid-eluting stents should be considered for future clinical and experimental testing.

\section{Acknowledgements}

This work was supported by a grant Reg. No. NV16-27653A (Czech Health Research Council) - Prevention of esophageal strictures after endoscopic resection or dissection of early esophageal neoplasia-experimental study.

Competing interests

The authors declare that they have no conflict of interest.

References

[1] Pimentel-Nunes P, Dinis-Ribeiro M, Ponchon T et al. Endoscopic submucosal issection: European Society of Gastrointestinal Endoscopy (ESGE) Guideline. Endoscopy 2015; 47: 829-854

[2] Miwata T, Oka S, Tanaka S et al. Risk factors for esophageal stenosis after entire circumferential endoscopic submucosal dissection for superficial esophageal squamous cell carcinoma. Surg Endosc 2016; 30: 4049-4056

[3] Martínek J, Juhas S, Dolezel R et al. Prevention of esophageal strictures after circumferential endoscopic submucosal dissection. Minerva Chir 2018; 73: 394-409

[4] Oliveira JF, Moura EG, Bernardo WM et al. Prevention of esophageal stricture after endoscopic submucosal dissection: a systematic review and meta-analysis. Surg Endosc 2016; 30: 2779-2791

[5] Wen J, Lu Z, Yang Y et al. Preventing stricture formation by covered esophageal stent placement after endoscopic submucosal dissection for early esophageal cancer. Dig Dis Sci 2014; 59: 658-663

[6] Pauli EM, Schomisch SJ, Furlan JP et al. Biodegradable esophageal stent placement does not prevent high-grade stricture formation after circumferential mucosal resection in a porcine model. Surg Endosc 2012; 26: 3500-3508

[7] Zajicek R, Mandys V, Mestak O et al. Human keratinocyte growth and differentiation on acellular porcine dermal matrix in relation to wound healing potential. Sci World J 2012; 2012: 727352

[8] Matoušková E, Mestak $\mathrm{O}$. The effect of different biologic and biosynthetic wound covers on keratinocyte growth, stratification and differentiation in vitro. J Tissue Eng 2014; 5: 2041731414554966
[9] Zajicek R, Matouskova E, Broz L et al. New biological temporary skin cover Xe-Derma $(\mathbb{R})$ in the treatment of superficial scald burns in children. Burns 2011; 37: 333-337

[10] Fischer A, Bausch D, Baier P et al. Risk of biodegradable stent-induced hypergranulation causing re-stenosis of a gastric conduit after esophageal resection. Endoscopy 2012; 44: E125-126

[11] Saito Y, Tanaka T, Andoh A et al. Novel biodegradable stents for benign esophageal strictures following endoscopic submucosal dissection. Dig Dis Sci 2008; 53: 330-333

[12] Shi Q, Ju H, Yao LQ et al. Risk factors for postoperative stricture after endoscopic submucosal dissection for superficial esophageal carcinoma. Endoscopy 2014; 46: 640-644

[13] Yamaguchi N, Isomoto H, Nakayama T et al. Usefulness of oral prednisolone in the treatment of esophageal stricture after endoscopic submucosal dissection for superficial esophageal squamous cell carcinoma. Gastrointest Endosc 2011; 73: 1115-1121

[14] Shoji H, Yamaguchi N, Isomoto $\mathrm{H}$ et al. Oral prednisolone and triamcinolone injection for gastric stricture after endoscopic submucosal dissection. Ann Transl Med 2014; 2: 22

[15] Hashimoto S, Kobayashi M, Takeuchi M et al. The efficacy of endoscopic triamcinolone injection for the prevention of esophageal stricture after endoscopic submucosal dissection. Gastrointest Endosc 2011; 74: 1389-1393

[16] Takahashi H, Arimura Y, Okahara S et al. A randomized controlled trial of endoscopic steroid injection for prophylaxis of esophageal stenoses after extensive endoscopic submucosal dissection. BMC Gastroenterol 2015; 15: 1

[17] Hashimoto S, Mizuno KI, Takahashi K et al. Evaluating the effect of injecting triamcinolone acetonide in two sessions for preventing esophageal stricture after endoscopic submucosal dissection. Endosc Int Open 2019; 7: E764-E770

[18] Martinek J. How to prevent post-ESD esophageal stricture. Endosc Int Open 2019; 7: E771-E773

[19] Ye LP, Zheng HH, Mao XL et al. Complete circular endoscopic resection using submucosal tunnel technique combined with esophageal stent placement for circumferential superficial esophageal lesions. Surg Endosc 2016; 30: 1078-1085

[20] Han Y, Guo J, Sun S et al. Acellular dermal matrix for esophageal stricture prevention after endoscopic submucosal dissection in a porcine model. Gastrointest Endosc 2017; 86: 1160-1167

[21] Schomisch S], Yu L, Wu Y et al. Commercially available biological mesh does not prevent stricture after esophageal mucosectomy. Endoscopy 2014; 46: 144-148

[22] Barret M, Pratico CA, Camus M et al. Amniotic membrane grafts for the prevention of esophageal stricture after circumferential endoscopic submucosal dissection. PLoS One 2014; 9: e100236

[23] Li L, Linghu E, Chai $\mathrm{N}$ et al. Efficacy of triamcinolone-soaked polyglycolic acid sheet plus fully covered metal stent for preventing stricture formation after large esophageal endoscopic submucosal dissection. Dis Esophagus 2019; 32: doi:10.1093/dote/doy121 